\title{
Bandwidth-Enhanced Oversampling Successive Approximation Readout Technique for Low-Noise Power-Efficient MEMS Capacitive Accelerometer
}

\author{
Longjie Zhong, Student Member, IEEE, Jun Yang, Member, IEEE, Donglai Xu, Senior Member, IEEE, \\ Xinquan Lai, Member, IEEE
}

\begin{abstract}
Bandwidth-Enhanced Oversampling Successive Approximation (BE-OSA) readout technique is proposed in this paper to reduce the noise floor of readout circuit for MEMS capacitive accelerometer while achieving high power efficiency in terms of the figure of merit (FoM). Open-loop structure has been widely used in MEMS capacitive accelerometer for the internet of things (IoT) applications due to its low power consumption. However, in the open-loop accelerometer, the capacitance variation of sensing element is limited to femto-farad level in order to overcome nonlinearity. As a result, the thermal noise from parasitic capacitance becomes significant. The ability of the readout circuit dealing with thermal noise is determined by the front-end switched-capacitor capacitance-to-voltage convertor (SC CVC) rather than the back-end ADC. To reduces the noise floor, traditional oversampling method increases the sampling frequency of SC CVC by increasing transconductance of amplifier, but this leads to low power efficiency. In this work, the BE-OSA technique provides a high power efficiency method as it increases the sampling frequency of SC CVC without increasing the transconductance of amplifier. The SC CVC based on BE-OSA technique is demonstrated in a readout circuit fabricated by a commercial $0.18 \mu \mathrm{m}$ BCD process and tested with a femto-farad MEMS accelerometer. The measurement results show that compared to the readout circuit without using BE-OSA technique, the readout circuit using BE-OSA reduces the noise floor from 2.5aF $/ \sqrt{ } \mathrm{Hz}$ to $0.9 \mathrm{aF} / \sqrt{\mathrm{Hz}}$. Compared with the other similar works, the proposed readout circuit achieves the best power efficiency in terms of both the absolute power efficiency $\left(F_{O} M_{2}=243 \mathrm{JJ}\right)$ among the switched-capacitor readout circuits and the relative power efficiency $\left(\mathrm{FoM}_{3}=0.14\right)$.
\end{abstract}

Index Terms-Capacitive sensor, readout circuit, sensor interface, MEMS accelerometer, parasitic capacitance, low noise, low power, power efficient, switched-capacitor.

This work was supported in part by the National Natural Science Foundation of China (NSFC) under Grant 61834002, and in part by the National Key Research and Development Program of China under Grant 2018YFB2202001. L. Zhong, J. Yang are with the National ASIC Research Center, Southeast University, Nanjing 210096, China. (J. Yang is the corresponding author, email: dragon@seu.edu.cn).

D. Xu is with the School of Science and Engineering, Teesside University, Middlesbrough TS1 3BA, U.K., and also with the School of Electrical and Electronic Engineering, Wuhan Polytechnic University, Wuhan 430048, China.

X. Lai is with the School of Electronics, Xidian University, Xi'an 710071, China.

\section{INTRODUCTION}

$\mathrm{M}$ EMS (Micro-electromechanical Systems) capacitive accelerometers play a fundamental part in a wide range of monitoring systems for health care applications [1] and internet of things (IoT) applications [2]. In these applications, sensors are powered by battery, therefore low power consumption and high power efficiency are required to extend battery life [3][4]. To achieve low power consumption, the readout circuits of MEMS accelerometers are designed with open-loop structure (e.g., charge control readout [5][6] and voltage control readout [5][7]-[9]) rather than close-loop one (e.g., force feedback readout [10]-[12]). Compared with the charge control readout structure, the voltage control readout structure offers lower cost. This is because the voltage control readout structure requires only two micromechanical sensing elements to implement fully differential structure while the charge control readout structure requires four [6][7]. However, the main issue of the voltage control readout structure is that its nonlinearity increases with the increase of capacitance variation of sensing element. To suppress the nonlinearity to an acceptable level, the capacitance variation has to be limited to several femto-farad level for an acceptable die cost [3]. But, the fact that the femto-farad level capacitance variation of sensing element is much lower than the parasitic capacitance of sensing electrodes which is at pico-farad-level results in two significant problems: 1) gain accuracy deterioration [13][14] and 2) signalto-noise ratio (SNR) deterioration [15]-[17]. These are the main challenges for the design of power efficient open-loop MEMS capacitive accelerometers.

The gain accuracy deterioration is caused by signal charge loss due to parasitic-induced gain error, which can be alleviated by three methods. The first method is simply enhancing the gain of pre-amplifier [18] to reduce the signal charge loss. The second method is use of negative impedance (negative- $R$ [19] or negative-C [20]) to compensate the signal charge loss. The third method is to calibrate/correct the gain error with the assistance of the digital circuits [21][22] or the switchedcapacitor circuits, such as the oversampling successive approximation (OSA) readout technique [9], correlated double sampling technique [23][24] and correlated level shifting technique [25]-[27], etc. This method is able to provide scaling- 


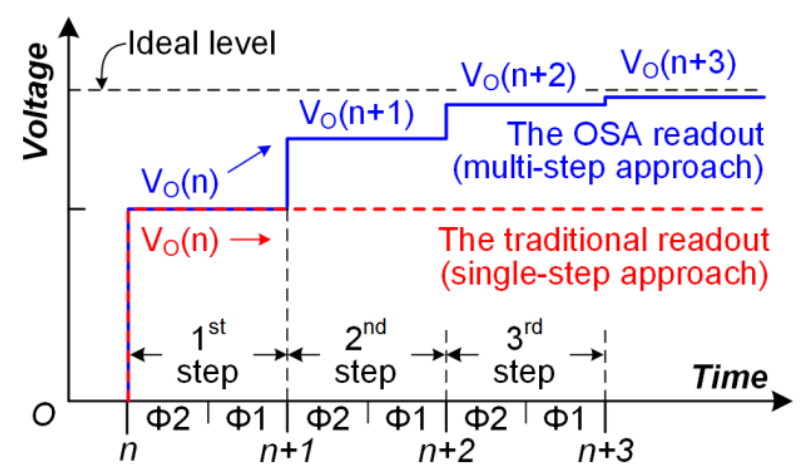

Fig. 1. Concept of OSA readout technique.

friendly solution in modern nanometer CMOS process and it is therefore very popular [28]. The switched-capacitor assisted techniques provide higher power efficiency in capacitive sensor readout circuits than the digital assisted techniques do, as the switched-capacitor assisted techniques are analog-assisted techniques, which do not need power hungry ADC/DAC to interface the assisting circuits with pre-amplifier [29]. Among the switched-capacitor assisted techniques, the OSA readout technique stands out for its insensitivity to parasitic-induced gain error [9]. The basic operation of OSA readout technique will be presented later. The kernel concept of the OSA readout technique is to approach the final output level of SC CVC by multiple steps, rather than traditional single step, as shown in Fig.1. In this way, the gain requirement of pre-amplifier is relaxed and the gain error is therefore minimized.

The SNR deterioration is caused by parasitic-induced noise charge. The existing solutions to this problem are the oversampling method [30], the wideband feedback control [31] [32], the feed forward cancellation [33][34], the holistic noisepower optimization [17] and the parasitic capacitance isolation stage [8] for switched-capacitor readout circuits and negative capacitance cancellation [35] for continuous-time readout circuits. However, these methods are not sufficiently power efficient. In this paper, a "bandwidth-enhanced oversampling successive approximation (BE-OSA)" readout technique is proposed for the switched-capacitor readout circuit to reduce the parasitic-induced noise while achieving high power efficiency. The proposed BE-OSA readout technique in this paper is an advanced version of the reported OSA readout technique [9]. The OSA readout technique focuses on reducing gain accuracy deterioration due to parasitic capacitance, while the BE-OSA readout technique focuses on reducing the SNR deterioration due to parasitic capacitance.

The rest of this paper is organized as follows. In section II, the noise performance of a typical open-loop readout circuit for MEMS accelerometer is discussed. In section III, BE-OSA readout circuit is proposed. In section IV, the physical verification and measurement results are presented. The conclusions are then drawn in section $\mathrm{V}$.

\section{NOISE PERFORMANCE OF READOUT CIRCUIT}

For the open-loop readout circuit with significant input parasitic capacitance, the trade-off between noise and power is determined by front-end switched-capacitor capacitance-to

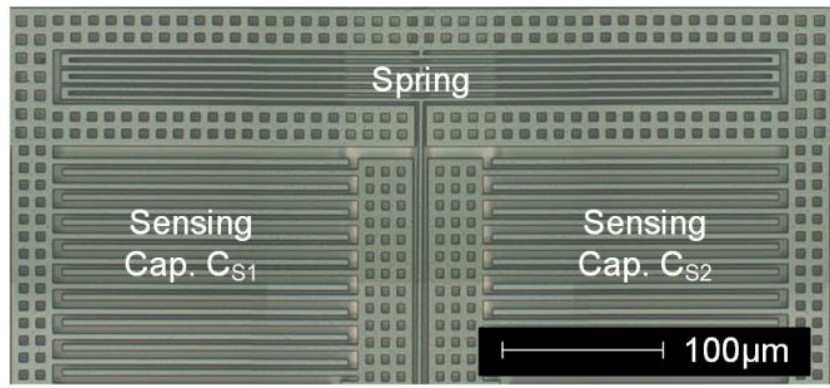

(a)

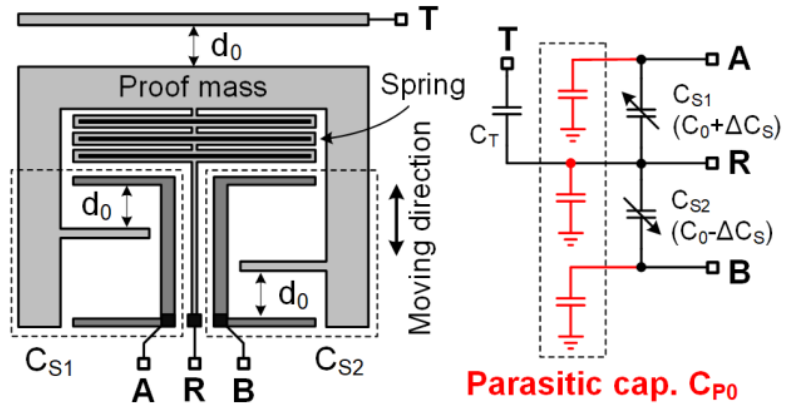

(b)

Fig. 2. Sensing element of a typical MEMS capacitive accelerometer. (a) Photograph. (b) Diagram.

voltage convertor (SC CVC) rather than back-end $\mathrm{ADC}$ [15][17]. In this section, the sensor structure and SC CVC noise performance are analyzed in detail.

\section{A. Sensor structure}

Fig. 2 shows the sensing element in a typical open-loop MEMS capacitive accelerometer. The parasitic capacitance $C_{P 0}$ (typically $3 \mathrm{pF}$ ) from the sensing electrodes $\mathrm{A}, \mathrm{B}$ and $\mathrm{R}$ to the ground is introduced by MEMS device $(1 \mathrm{pF})$, electro-static discharge protector $(1.5 \mathrm{pF})$ and bonding $\mathrm{pad}(0.5 \mathrm{pF})$. The sensing capacitances $C_{S 1}$ and $C_{S 2}$ are the parallel-plate capacitors formed by the stator plates (unmovable plates connected to the electrodes $\mathrm{A}$ and $\mathrm{B}$ ) and the rotor plates (movable plates on the proof mass connected to the electrode $\mathrm{R}$ via spring), which are expressed below [36],

$$
C_{S 1}=C_{0}\left(\frac{1}{1+\Delta d / d_{0}}\right), C_{S 2}=C_{0}\left(\frac{1}{1-\Delta d / d_{0}}\right)
$$

where $C_{0}$ is the rest capacitance of sensing capacitance, $d_{0}$ is the rest distance between a pair of rotor plate and stator plate, $\Delta d$ is the rotor plate displacement which is in proportion to applied acceleration signal. Equation (1) is a nonlinear function which is undesirable for acceleration measurement. Hence, the linear function (2) is used to approximate the equation (1),

$$
\begin{aligned}
& C_{S 1} \approx C_{0}\left(1-\Delta d / d_{0}\right)=C_{0}-\Delta C_{S} \\
& C_{S 2} \approx C_{0}\left(1+\Delta d / d_{0}\right)=C_{0}+\Delta C_{S}
\end{aligned}
$$

According to Taylor's theorem, only by limiting the displacement ratio $\Delta d / d_{0}$ to a small value (e.g., $3.2 \%$ ), can the high-order nonlinear approximation error of the equation (2) be limited to an acceptable level (e.g., <0.1\%). Thus, given the sensor's typical rest capacitor $C_{0}$ being $160 \mathrm{fF}$, the maximum capacitance variation $\Delta C_{S}$ will not exceed $5.0 \mathrm{fF}$ to achieve $0.1 \%$ 


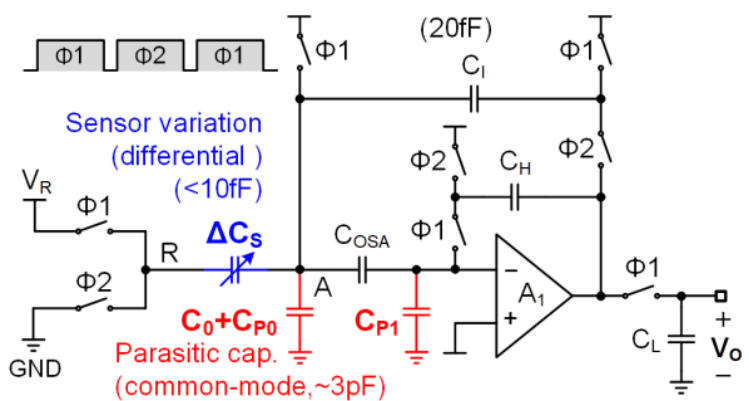

(a)

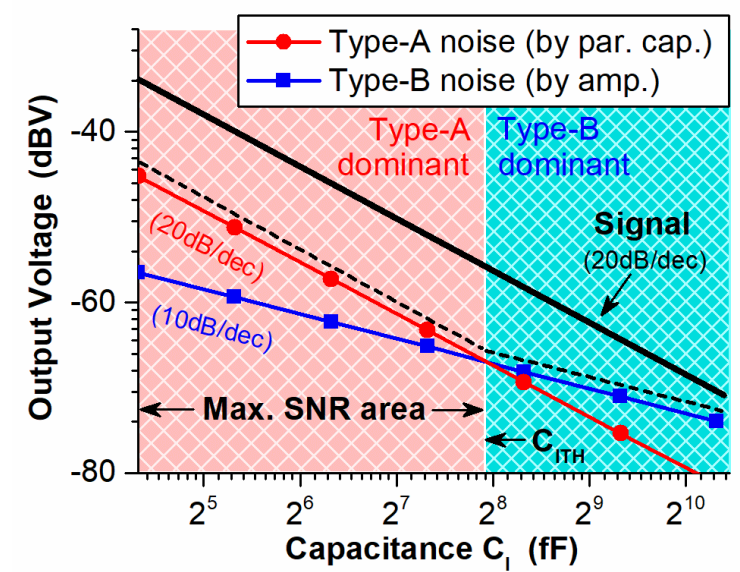

(a)

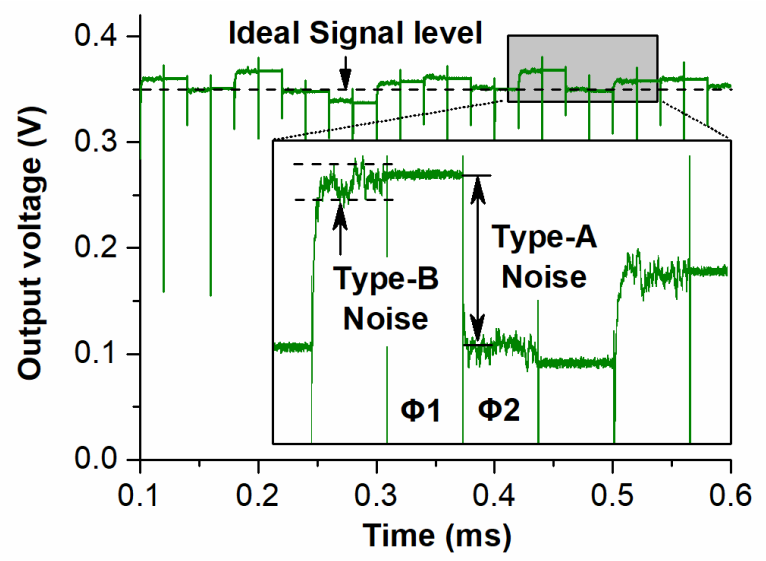

(b)

Fig. 3. Noise analysis of SC CVC. (a) Simplified single-end equivalent circuit model. (b) Relationship between dominant noise and capacitance $C_{I}$. (b) Typical transient output waveform of SC CVC when SNR is maximized. nonlinearity.

\section{B. SC CVC noise performance}

The simplified single-end equivalent circuit of the SC CVC based on OSA readout technique (OSA CVC in brief) used to readout the sensing element is shown in Fig. 3(a), where $C_{P 0}$ is the parasitic capacitance between sensing electrodes and ground, $C_{P 1}$ is the parasitic capacitance introduced by the input transistors of the pre-amplifier $A_{1}$. The rest capacitance $C_{0}$ of sensing element is also modelled as parasitic capacitance, as it does not contribute to any signal charge. The basic operation of OSA CVC is described as follows. In each step, the preamplifier $A_{1}$ holds the output level $V_{O}(n)$ via the feedback network composed of capacitor $C_{H}$ and $C_{O S A}$, so that the gain error term of $V_{O}(n)$ is sampled and will be cancelled in the next

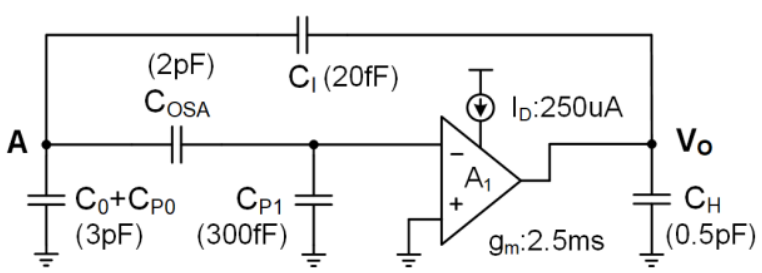

Fig. 4. Equivalent circuit model with typical component parameters of SC CVC during phase $\Phi 2$.

step by the capacitor $C_{O S A}$. As a result, the "new" gain error in the next step is produced by the increment value of the output in the step $\Delta V_{O}(n+1)$, rather than by the absolute value of the output in this step. Thus, the "new" gain error is smaller than the "old" gain error. Step by step, the gain error is diminished and reduces towards zero exponentially. This means that the gain error will be reduced to infinitesimal at cost of settling time. In this way, the gain requirement of the pre-amplifier $A 1$ used in the OSA CVC is relaxed and the gain error is therefore minimized.

1) SNR maximization. According to [30], there are two types of output noise power: the type-A noise $\overline{V_{N A}^{2}}$ from parasitic capacitance during the phase $\Phi 1$ and the type-B noise $\overline{V_{N B}^{2}}$ produced by the pre-amplifier $A_{1}$ during the phase $\Phi 2$. They are expressed as follows,

$$
\begin{gathered}
\overline{V_{N A}^{2}}=\frac{k T\left(C_{0}+C_{P 0}\right)}{C_{I}^{2}}+\frac{k T C_{P 1}}{C_{I}^{2}} \frac{1}{\beta_{2}^{2}} \\
\overline{V_{N B}^{2}}=\frac{1}{\beta_{1} \beta_{2}} \frac{k T}{C_{H}} \alpha ; V_{O}=\frac{\Delta C_{S}}{C_{I}} V_{R} \\
\beta_{1}=\frac{C_{I}}{C_{0}+C_{P 0}} ; \beta_{2}=\frac{C_{O S A}}{C_{O S A}+C_{P 1}}
\end{gathered}
$$

where $\alpha$ is a constant coefficient with a typical value of 1.5 [30], $V_{R}$ is reference voltage, $C_{H}$ is load capacitor during phase $\Phi 2$. Equation (3) indicates an important fact that with the decrease of the capacitance $C_{I}$, the output signal voltage $V_{O}$ of SC CVC increases at a rate of $20 \mathrm{~dB} / \mathrm{dec}$ and the output type-A noise voltage $\overline{V_{N A}}$ increases at a rate of $20 \mathrm{~dB} / \mathrm{dec}$ while the output type-B noise voltage $\overline{V_{N B}}$ increases at a rate of $10 \mathrm{~dB} / \mathrm{dec}$. As a result, the SNR will be maximized when the capacitance $C_{I}$ is minimized and the type-A noise dominates, as shown in Fig. 3(b). The threshold of the capacitance $C_{I}$ to maximize SNR is,

$$
C_{I T H}=\frac{C_{H}}{\alpha}\left[\beta_{2}+\frac{C_{P 1}}{\beta_{2}\left(C_{0}+C_{P 0}\right)}\right]
$$

When $C_{I} \ll C_{I T H}$, the noise from amplifier (type-B) is negligible, as shown in Fig. 3(c). In this situation, the power consumption of the pre-amplifier $A_{1}$ is no longer determined by its noise performance requirement but by its bandwidth requirement.

2) Noise floor and bandwidth deterioration. As mentioned in the equation (4), the capacitance $C_{I}$ should be minimized to maximize SNR. Besides, minimizing the capacitance $C_{I}$ can maximize the sensitivity of SC CVC according to the equation (3), which can further relax the accuracy requirement of the back-end ADC. However, reducing the capacitance $C_{I}$ leads to 


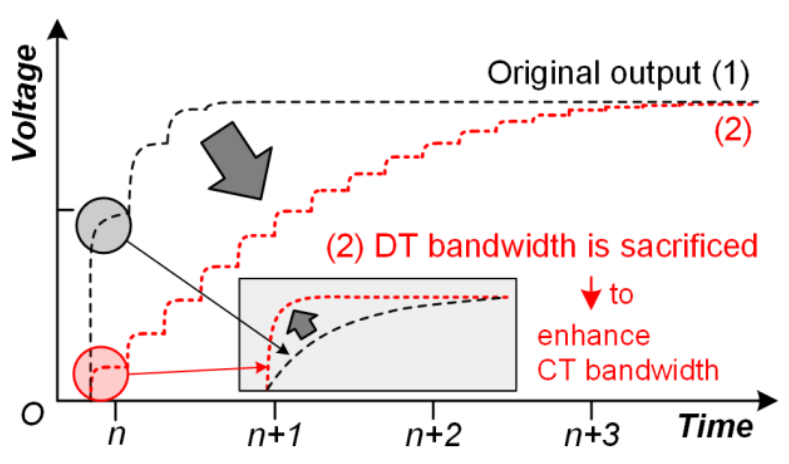

(a)

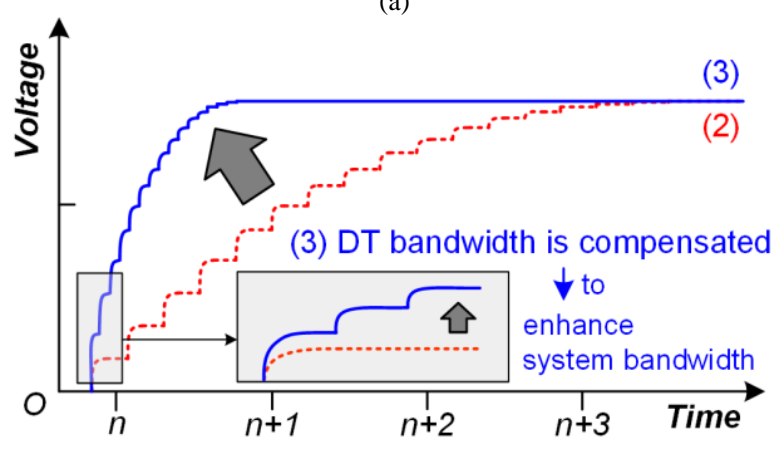

(b)

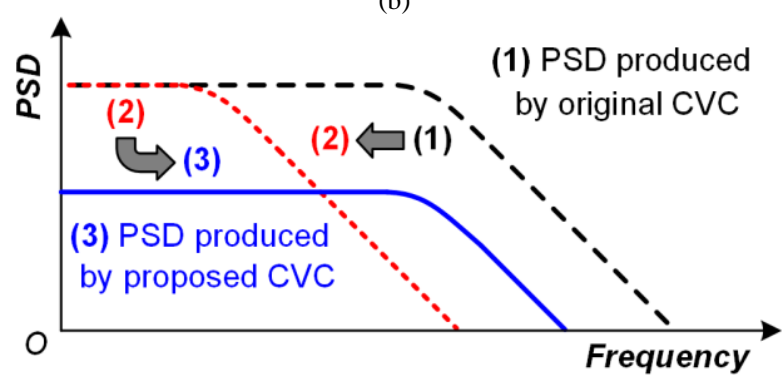

(c)

Fig. 5. Concept of BE-OAS technique to reduce noise. (a) DT bandwidth is sacrificed to improve CT bandwidth. (b) Sampling frequency is increased and DT bandwidth is enhanced to compensate system bandwidth. (c) Noise PSD.

reduction of the feedback coefficient $\beta_{1}$ according to the equation (3). And the reduction of $\beta_{1}$ lastly result in reduction of the SC CVC's loop bandwidth $B W_{C T}$ that can be expressed below,

$$
B W_{C T}=\beta_{1} \beta_{2} \frac{g_{m}}{C_{H}}
$$

where $g_{m}$ is the transconductance of the pre-amplifier $A_{1}$. Equation (5) is derived from the circuit model in Fig. 4.

Further, reduction of the bandwidth $B W_{C T}$ will lead to reduction of the maximum sampling frequency $f_{\text {smax }}$ for a given settling error, as shown by the following expression [17],

$$
e^{-\frac{B W_{C T}}{2 f_{\text {smax }}}} \leq \text { Settlig error }
$$

The reduction of the maximum sampling frequency $f_{\text {smax }}$ eventually leads to increase of the noise floor that is due to noise aliasing effect and is measured here by power spectrum density (PSD) [30], as shown below,

$$
P S D=\frac{2}{\pi} \frac{\overline{V_{N A}^{2}}}{f_{\text {smax }}}
$$

Thus, although a small value of $C_{I}$ will bring high SNR and high sensitivity, the noise floor will be high as well due to reduced sampling frequency. If high sampling frequency is used to reduce the noise floor, large power will be consumed. For example, to support a sampling frequency $f_{s}$ of $1 \mathrm{MHz}$ with $0.1 \%$ settling error, the bandwidth $B W_{C T}$ should be at least $13.8 \mathrm{MHz}$ according to the equation (6). Given $C_{I}$ 's value being $20 \mathrm{fF}$, as shown in Fig. 4, the value of $\beta_{1}$ is as small as 0.007 . As a result, $g_{m}$ should be as large as $2.5 \mathrm{~ms}$ according to the expression (5), which requires a typical current supply $I_{\mathrm{D}}$ of $250 \mu \mathrm{A}$. In practice, this level of current consumes too much power and is therefore too large to be acceptable in IoT applications [3][4].

\section{BANDWIDTH-ENHANCED OVERSAMPLING SUCCESSIVE APPROXIMATION (BE-OSA) READOUT TECHNIQUE}

The BE-OSA technique is presented in this section. It increases the sampling frequency of SC CVC without increasing the gain-bandwidth product of pre-amplifier, thus reducing the noise floor without sacrificing power efficiency. For the clarity of explanation, the following three bandwidth terms are used.

1) Continuous-Time (CT) bandwidth, B $\boldsymbol{W}_{\boldsymbol{C T}}$. The $\mathrm{CT}$ bandwidth is the closed loop bandwidth of an amplifier, whose value is dependent on $g_{m} / C$ constant and feedback coefficient [37]. In this work, the CT bandwidth determines the maximum value of CVC's sampling frequency $f_{S}$.

2) Discrete-Time (DT) bandwidth, $\boldsymbol{B} \boldsymbol{W}_{\boldsymbol{D T}}$. The DT bandwidth is the system bandwidth with normalized sampling frequency $f_{S}$, whose value is dependent on the pole of $\mathrm{Z}$ transfunction of the system. In this work, the DT bandwidth determines the number of steps/periods that CVC needs to settle down.

3) System bandwidth, $\boldsymbol{B} \boldsymbol{W}_{\boldsymbol{S Y}}$. The system bandwidth refers to the "- $3 \mathrm{~dB}$ bandwidth" of the system, whose value is dependent on the product of $B W_{D T}$ and $f_{S}$ [37]. In this work, the system bandwidth determines the total time that SC CVC needs to settle down.

\section{A. Principle}

The basic concept of the BE-OSA readout technique is shown in Fig. 5, which includes two stages to achieve power efficient noise floor reduction.

At the stage one, DT bandwidth is sacrificed for CT bandwidth, as shown in Fig. 5(a). Compared with the output of the original OSA CVC (Fig. 2(b)), the output of the BE-OSA CVC takes more steps (sacrificing DT bandwidth) to reach the same final output level, hence the gain requirement of each step is reduced. As pre-amplifier's gain-bandwidth product is constant, the CT bandwidth in each step is therefore enhanced without increasing the pre-amplifier's power consumption.

At the stage two, sampling frequency is increased and DT bandwidth is compensated, as shown in Fig. 5(b). Since the CT bandwidth is improved, the maximum sampling frequency can 


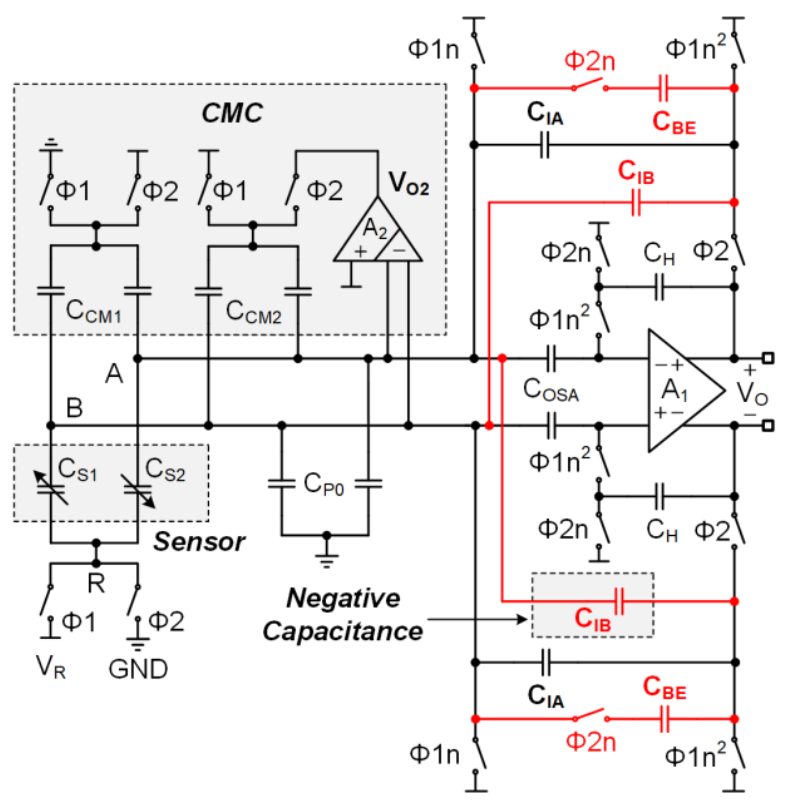

(a)

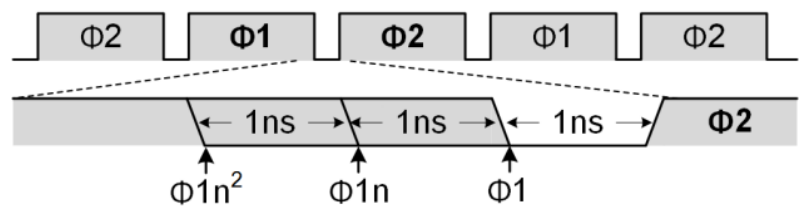

(b)

Fig. 6. BE-OSA CVC with negative capacitance $C_{I B}$ and CT bandwidth enhancement capacitor $C_{B E}$. (a) Schematic. (b) Clock diagram.

be increased. As a result, the noise floor is reduced. A DT bandwidth compensation circuit is also employed at this stage to compensate the DT bandwidth sacrificed at the stage one, in order to avoid the loss of system bandwidth.

The noise PSDs of each step are shown in Fig. 5(c). Compared with the PSD produced by the original $\mathrm{CVC}$, the PSD produced by the proposed BE-OSA CVC is reduced without losing system bandwidth.

The key circuits to implement BE-OSA CVC are: 1) sensitivity boost circuit, 2) CT bandwidth enhancement circuit and 3) DT bandwidth compensation circuit. The other circuits, such as common-mode charge controller (CMC), etc., can be found in [9] and they are not discussed here.

\section{B. Circuit-I: sensitivity boost}

As mentioned in section II, the capacitance $C_{I}$ should be minimized to maximize SNR and sensitivity. However, due to physical size limitation in manufacturing process, small capacitance sometimes cannot be directly acquired. So the negative capacitance $C_{I B}$ is employed here to produce an equivalent small value capacitor $C_{I}$, as shown in Fig. 6(a). For example, if the expected value of $C_{I}$ is $20 \mathrm{fF}$ while the minimum capacitance possible is $30 \mathrm{fF}$, then $C_{I}$ of $20 \mathrm{fF}$ can be produced by $C_{I A}$ of $50 \mathrm{fF}$ and $C_{I B}$ of - $30 \mathrm{fF}$. The output voltage is,

$$
V_{O}=\frac{2 V_{R}}{C_{I A}+C_{I B}} \Delta C_{S}=\frac{2 V_{R}}{C_{I}} \Delta C_{S}
$$

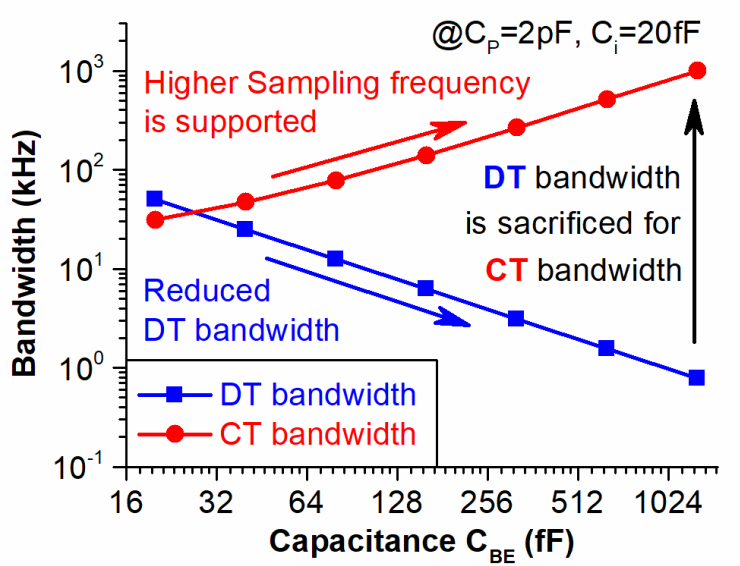

Fig. 7. Bandwidth change with the change of $C_{B E}$

\section{Circuit-II: CT bandwidth enhancement}

The CT bandwidth is enhanced by the capacitor $C_{B E}$ in Fig.6(a), which plays two important roles. The first role is the CT auxiliary feedback path enhancing the feedback coefficient $\beta_{1}$, hence increasing the CT bandwidth. With $C_{B E}$, the CT bandwidth becomes,

$$
\begin{gathered}
B W_{C T}^{\prime}=\beta_{1}^{\prime} \beta_{2} \frac{g_{m}}{C_{H}+C_{L}} \\
\beta_{1}^{\prime}=\frac{C_{I}+C_{B E}}{C_{I}+C_{P 0}+C_{B E}} ; \beta_{2}=\frac{C_{O S A}}{C_{O S A}+C_{P 1}}
\end{gathered}
$$

where $\beta_{1}^{\prime}$ is the feedback coefficient enhanced by $C_{B E}$. The second role of $C_{B E}$ is an integrator which sacrifices the DT bandwidth. The BE-OSA CVC's transfer function and the DT bandwidth are,

$$
\begin{gathered}
H_{1}(z)=\left(z-p_{0}\right)^{-1} \\
B W_{D T}=\frac{2}{\pi} \tan ^{-1}\left(\frac{1-p_{0}}{1+p_{0}}\right) ; p_{0}=\frac{C_{B E}}{C_{B E}+C_{I}}
\end{gathered}
$$

Equations (9) and (10) show that when $C_{B E}$ is smaller than $C_{P 0}$, with increase of the value of the capacitor $C_{B E}$, the CT bandwidth increases at a rate of $+20 \mathrm{~dB} / \mathrm{dec}$ while the DT bandwidth decreases at a rate of $-20 \mathrm{~dB} / \mathrm{dec}$, as shown in Fig. 7 . Therefore, the CT bandwidth can be improved at the cost of the DT bandwidth rather than sacrificing power consumption.

\section{Circuit-III: DT bandwidth compensation}

In order to avoid the loss of system bandwidth due to sacrificing DT bandwidth, the compensation circuit composed of the capacitor $C_{D E}$ and the amplifier $A_{3}$ is added, as shown in Fig. 8(a). Notably, the additional power consumption introduced by the amplifier $A_{3}$ is negligible compared to that of pre-amplifier $A_{1}$. This is because the feedback capacitor $C_{f}$ can be set to a large value to minimize the $g_{m}$ requirement of $A_{3}$.

The rationale of the DT bandwidth compensation circuit is to speed up the charging process of the $\mathrm{CT}$ bandwidth enhancement capacitor $C_{B E}$ via the capacitor $C_{D E}$. The larger the value of $C_{D E}$ is, the faster $C_{B E}$ is charged, and then the more DT bandwidth can be compensated. However, if $C_{D E}$ is too large, 


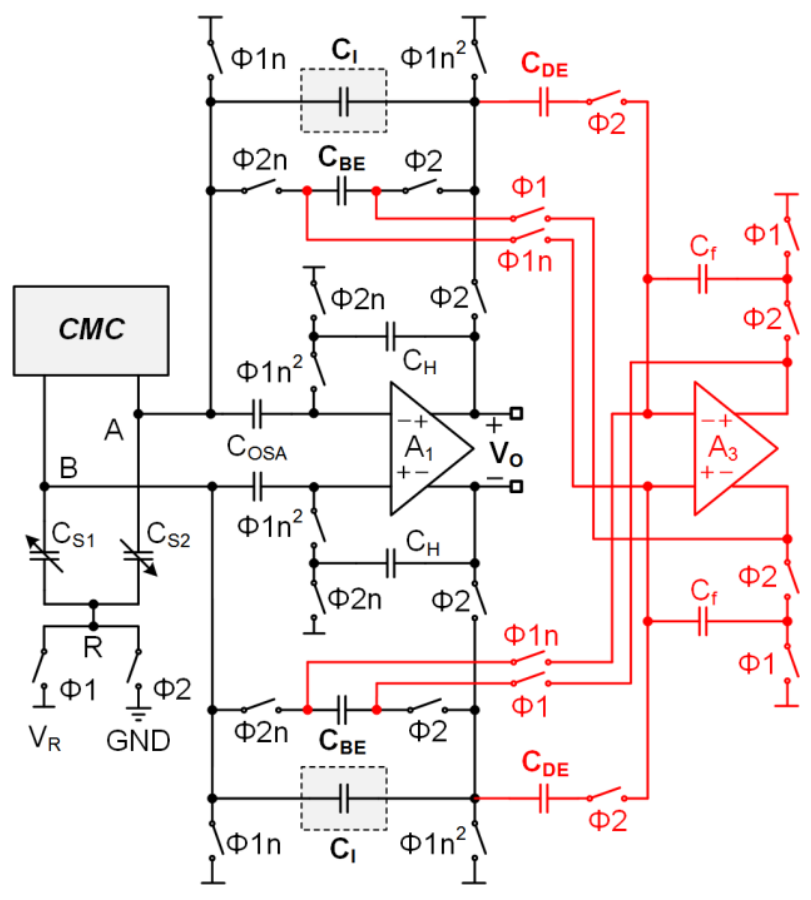

(a)

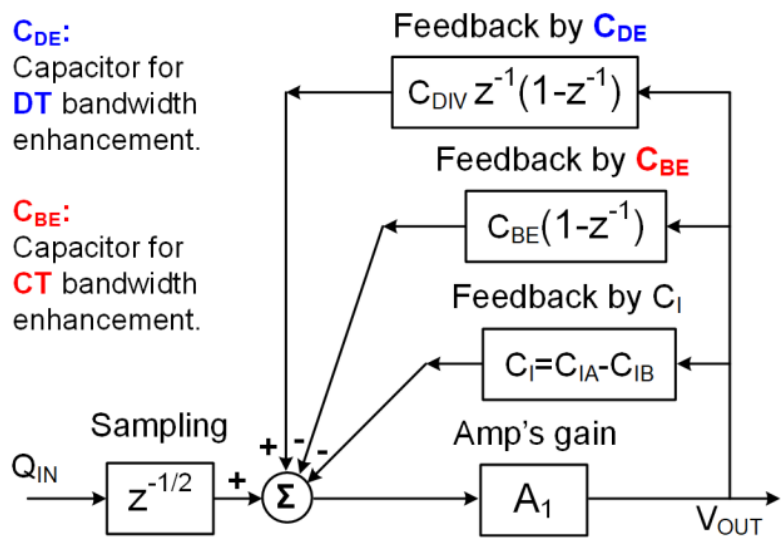

(b)

Fig. 8. Complete circuit implementation of BE-OSA CVC. (a) Schematic. (b) Signal diagram.

the system will become unstable. According to the signal flow diagram shown in Fig. 8(b), the system transfer function is,

$$
\begin{gathered}
H_{2}(z)=\frac{z^{2}}{z^{2}-a z+b} \\
a=\frac{C_{B E}+C_{D E}}{C_{B E}+C_{I}}, b=\frac{C_{D E}}{C_{B E}+C_{I}}
\end{gathered}
$$

To guarantee system's stability, the condition below has to be satisfied,

$$
a^{2} \geq 4 b,\left|\frac{a \mp \sqrt{a^{2}-4 b^{2}}}{2}\right| \leq 1
$$

Then the maximum value of the capacitor $C_{D E}$ can be acquired by solving (17),

$$
C_{D E}=C_{B E}-2 C_{I}\left(\sqrt{1+C_{B E} / C_{I}}-1\right)
$$

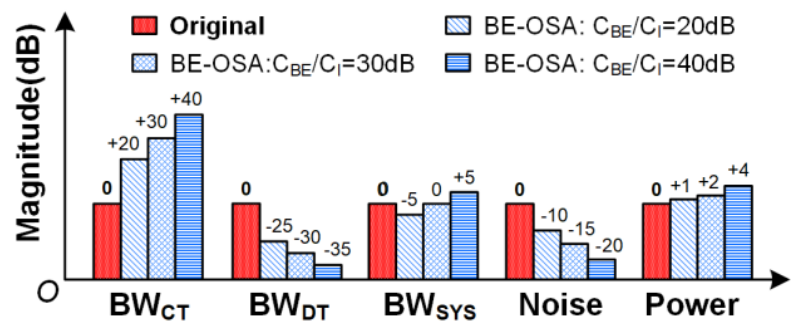

Fig. 9. Comparison of bandwidth, noise performance and power consumption between original CVC and BE-OSA CVC.

By applying the equation (13) to the equation (11), the new pole is

$$
p_{0}^{\prime}=\frac{1}{2} \frac{C_{B E}+C_{D E}}{C_{B E}+C_{I}}
$$

It can be seen from the equations (10), (13) and (14) that the value of the new pole $p_{0}^{\prime}$ is smaller than that of the original pole $p_{0}$. Thus, the new pole provides higher DT bandwidth.

The system bandwidth $B W_{S Y S}$ is determined by the maximum sampling frequency $f_{\text {smax }}$ and the DT bandwidth $B W_{D T}$,

$$
B W_{S Y S}=2 \pi f_{\text {Smax }} B W_{D T}
$$

An intuitive illustration of the BE-OSA technique's effect is shown in Fig.9. According to the expression (14), with increase of $C_{B E}$, the CT bandwidth is increased. This leads to increase of sampling frequency and reduction of noise floor. Furthermore, according to the equations (10), (14) and (15), the amount of the sacrificed DT bandwidth is less than that of the increased CT bandwidth. Thus, the overall system bandwidth loss is negligible. The slight power increment is due to the use of the amplifier $A_{3}$ and the switched-capacitor network with increased sampling frequency.

\section{PHYSICAL VERIFICATION}

The BE-OSA CVC described above is demonstrated in a readout circuit fabricated by a commercial $0.18 \mu \mathrm{m}$ BCD process. The readout IC is tested with a femto-farad 3-axis MEMS capacitive accelerometer (only one axis is measured), as shown in Fig. 10(a) and Fig. 10(b). The readout IC integrates BE-OSA CVC, CMC, calibration circuits, references, clock generator and buffers, as shown in the layout in Fig. 10(c). The on-chip clock generator includes an oscillator to generate clock source and a non-overlapping clock generator to provide nonoverlapping clock phases. When the readout circuit is in the test mode, the output frequency of the clock generator is synchronized by an outside clock source. The pre-amplifier $A_{1}$ used in SC CVC is a traditional fold-cascade amplifier with 70dB gain, 10MHz GBW@2pF capacitive load, 16nV/ $\mathrm{JHz}$ input equivalent noise, 200fF input parasitic capacitance and $30 \mu \mathrm{A}$ current consumption. The amplifier $A_{2}$ has a $10 \mu \mathrm{A}$ current consumption and a $100 \mathrm{fF}$ input parasitic capacitance. The amplifier $A_{3}$ has a $3 \mu \mathrm{A}$ current consumption.

A $200 \mathrm{~Hz}$ test excitation $V_{T E S T}=V_{O S}+A_{0} \sin \left(\omega_{0} t\right)$ generated by the spectrum analyzer (APX525) is applied to the 


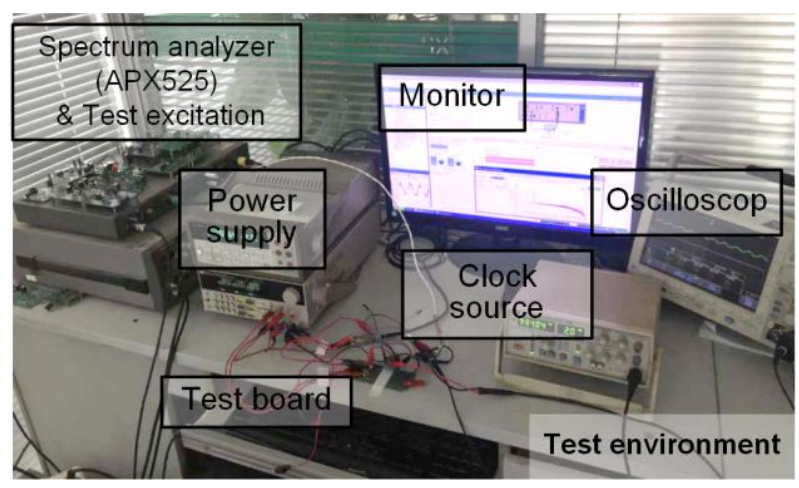

(a)

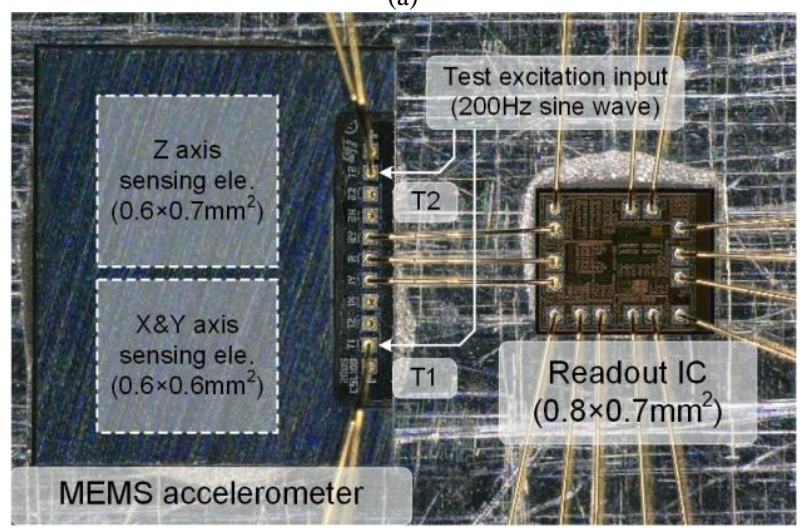

(b)

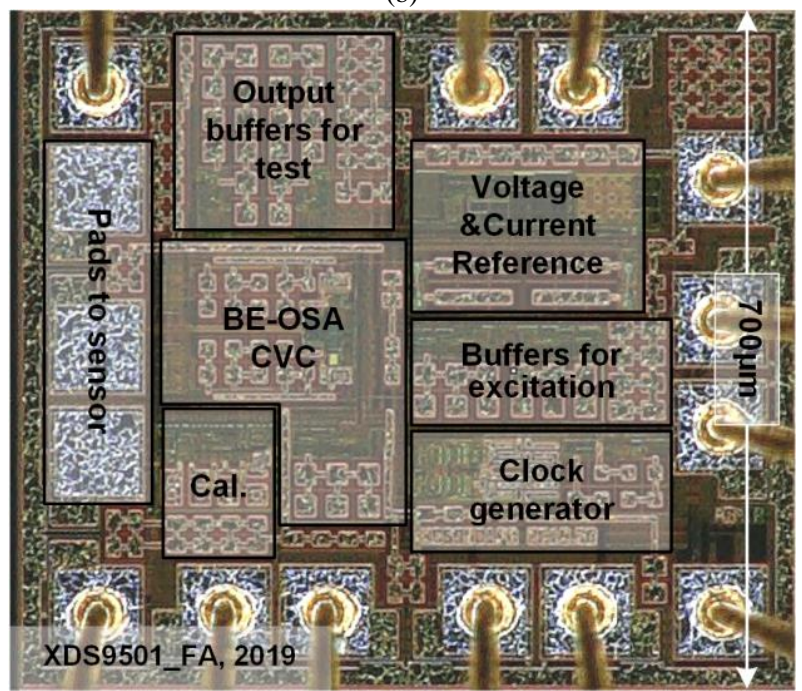

(c)

Fig. 10. Readout chip based on BE-OSA technique tested with a femto-farad MEMS accelerometer. (a) Test environment. (b) Photograph of the chip bonded with the MEMS accelerometer. (c) Photograph of the chip.

test electrode $\mathrm{T}$ (shown in Fig.2) of sensing element. An electrostatic force $F_{T E S T}$ is then produced by the test electrode $\mathrm{T}$ to drive the proof mass of the sensing element. The electrostatic force can simulate input acceleration signal, which is expressed as,

$$
\begin{aligned}
F_{T E S T}=\frac{1}{2} \frac{C_{T}}{d_{0}} & {\left[(1-D)\left(V_{O S}+A_{0} \sin \left(\omega_{0} t\right)\right)^{2}\right.} \\
& \left.+D\left(V_{R}-V_{O S}-A_{0} \sin \left(\omega_{0} t\right)\right)^{2}\right]
\end{aligned}
$$

where $d_{0}$ have been defined in the equation (1), $C_{T}$ is the capacitance between the test electrode T and the proof mass, $D$ is the duty circle of phase $\Phi 1$. Given the conditions $D=50 \%$

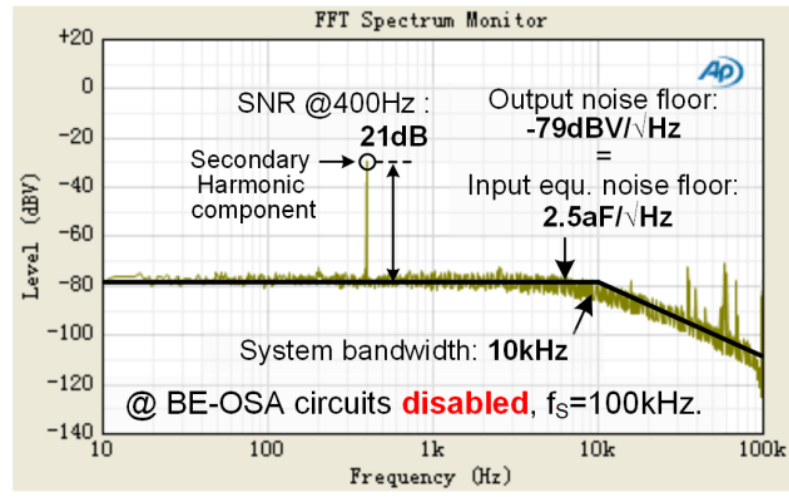

(a)

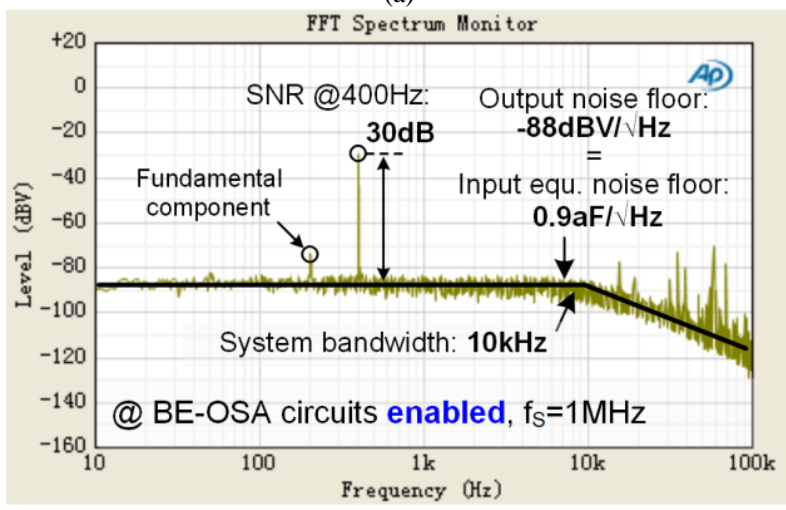

(b)

Fig.11. Measurement result of output PSD with $128 \mathrm{k}$ points FFT. (a) BE-OSA circuits are disabled. (b) BE-OSA circuits are enabled.

and $V_{O S}=0.5 V_{R}$, the equation (16) is simplified as,

$$
F_{T E S T}=\frac{1}{4} \frac{C_{T}}{d_{0}}\left[\frac{1}{2} V_{R}^{2}+A_{0}^{2}\left(1-\cos \left(2 \omega_{0} t\right)\right]\right.
$$

Equation (17) shows that there is no fundamental component and only secondary harmonic component $(400 \mathrm{~Hz})$ exists in the sensor's response. This is also illustrated in the output PSD in Fig. 11. Therefore, only the secondary harmonic component is used to calculate SNR or noise floor.

A comparison experiment between with and without BEOSA technique is conducted. Firstly, when the BE-OSA circuit blocks (i.e., circuit-II and circuit-III) are disabled, the singleend output noise floor is $-79 \mathrm{dBV} / \sqrt{\mathrm{Hz}}$ (equal to an input equivalent noise floor of $2.5 \mathrm{aF} / \sqrt{\mathrm{Hz}}$ ) and the $\mathrm{SNR}$ at $400 \mathrm{~Hz}$ is 21dB, as shown in Fig. 11(a). Then, when the BE-OSA circuit blocks are enabled, the sampling frequency can be improved from $100 \mathrm{kHz}$ to $1 \mathrm{MHz}$, the output noise floor is reduced to $88 \mathrm{dBV} / \sqrt{\mathrm{Hz}}$ (equal to an input equivalent noise floor of $0.9 \mathrm{aF} / \sqrt{\mathrm{Hz}}$ ) and the SNR is improved to $30 \mathrm{~dB}$, as shown in Fig. 11(b). The current consumption from power supply is measured by a $200 \Omega$ series shunt resistor and a commercial low noise amplifier LT1997-1. The measurement results are shown in Fig. 12(a). When the BE-OSA circuit blocks are disabled, the total current consumption of the readout IC is $256 \mu \mathrm{A}$. When the BEOSA circuit blocks are enabled, the total current consumption increases from $259 \mu \mathrm{A}$ to $262 \mu \mathrm{A}$ with the sampling frequency increasing from $0.1 \mathrm{MHz}$ to $1 \mathrm{MHz}$. So the increment of the total current consumption by using BE-OSA circuits at $1 \mathrm{MHz}$ sampling frequency is $6 \mu \mathrm{A}(262 \mu \mathrm{A}-256 \mu \mathrm{A})$. The current consumptions from each building block at $1 \mathrm{MHz}$ sampling 


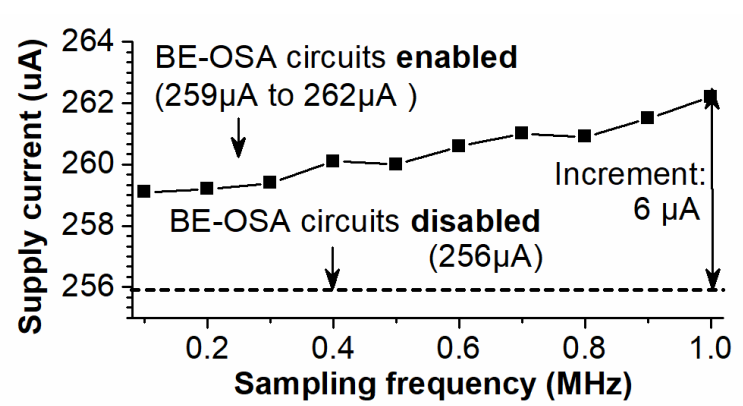

(a)
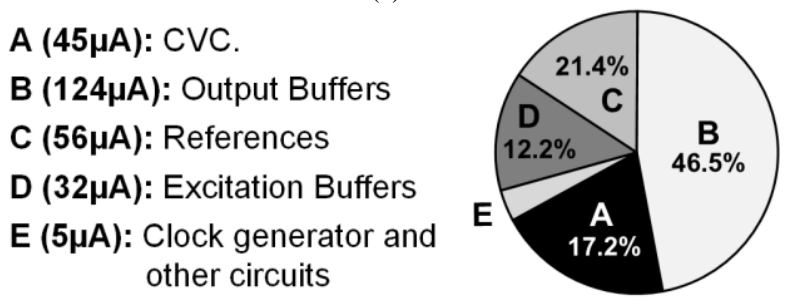

(b)

Fig.12. Measurement results of current consumption. (a) Current of whole chip. (b) Current consumption of each building block.

frequency are shown in Fig. 12(b), where CVC consumes 17.2\% $(45 \mu \mathrm{A})$ of the total current. The increment of total current consumption $(6 \mu \mathrm{A})$ is mainly due to CVC. Thus, by employing the BE-OSA circuits, the current consumption of CVC increases by $1.2 \mathrm{~dB}$ from $39 \mu \mathrm{A}$ to $45 \mu \mathrm{A}$, but the noise floor is reduced by $9 \mathrm{~dB}(88 \mathrm{~dB}-79 \mathrm{~dB})$. The spectrum peak in high frequency range is due to deterministic interference rather than random noise.

The comparison between BE-OSA and other similar parasitic-induced noise reduction techniques for $\mathrm{CVC}$ is given in Table I. Generally, there are two main types of figure of merit (FoM) used to evaluate the power efficiency of MEMS accelerometer [11],

$$
\mathrm{FoM}_{1 A}[W \cdot F / H z]=\frac{\text { Power } \times \text { Noise floor }}{\sqrt{B W}}
$$

or [34],

$$
\mathrm{FoM}_{1 B}[W \cdot F / H z]=\frac{\text { Power }}{\text { Dynamic range } \times B w}
$$

The $F o M_{1 A}$ emphasizes on the current efficiency, i.e., to reduce the noise floor with as little increment of supply current as possible. The $F o M_{1 B}$ emphasizes on the voltage efficiency, i.e., to increase the signal range with as little increment of supply voltage as possible [38]. Since the purpose of this work is to improve the current efficiency/utilization, the $F_{O} M_{1 A}$ is preferred. However, the comparison only by $F_{O} M_{1 A}$ may not be true reflection of power efficiency, as $F_{O} M_{1 A}$ is defined for close-loop or large size accelerometers for industrial applications whose noise performance is not dominated by the parasitic capacitance. In open-loop or small size accelerometer for IoT applications, the output noise is significantly affected by the input parasitic capacitance which should be taken into account for the measurement of FoM [15]. Therefore, a modified FoM is introduced for fairer comparison, which is defined as,

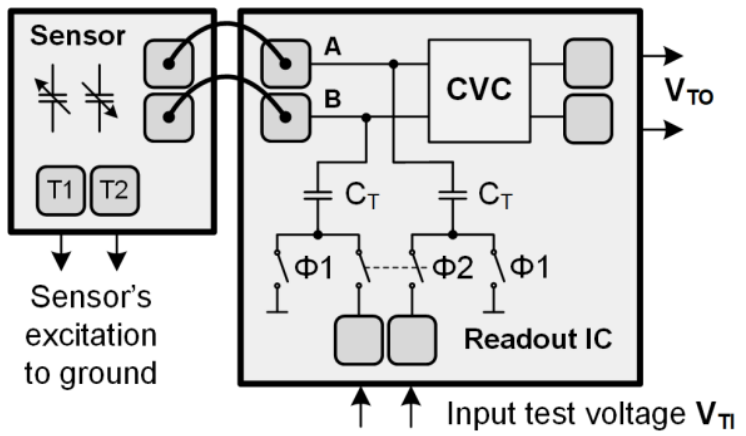

Fig.13. Measurement configuration of IC sensitivity.

$$
\mathrm{FoM}_{2}[J]=\frac{\text { Power } \times \text { Noise floor }}{C_{P} \times \sqrt{B w}}
$$

where $C_{P}$ is parasitic capacitance. Using $F_{O} M_{2}$, this work shows the best power efficiency among the switched-capacitor CVCs ([9] and [17]), as shown in Table I. The work [17] achieves the lowest absolute current consumption $(0.13 \mu \mathrm{A})$ but with poor power efficiency $(2,416 \mathrm{fJ})$. This is because the direct successive approximation capacitive-to-digital converter (SAR CDC) structure in [17] uses the capacitor digital-to-analog convertor (CDAC) as the feedback network which introduces additional Type-A noise charge [8]. The work [9] employs the traditional oversampling method and achieves the poorest power efficiency $(62,856 \mathrm{fJ})$. The continuous-time CVCs ([35][39][40]) shows averagely better FoM2 than that of switched-capacitor CVCs ([7], [9] and this work). This is because the basic continues-time operation is free from the noise aliasing effect introduced by sampling operation [30]. However, continuous-time operation loses the ability to settle out offset and flicker noise from per-amplifier and therefore surfers from serious flicker noise at low frequency range.

Furthermore, as noise reduction techniques lead to increase of power consumption, the relative FoM2 is adopted here to evaluate more accurately power efficiency. It is defined as,

$$
F_{O M} M_{3}=\frac{20 \log (\Delta P)}{20 \log \left(\triangle F o M_{2}\right)}
$$

where $\Delta \mathrm{FoM}_{2}$ is the percentage of $\mathrm{FoM}_{2}$ improvement, and $\Delta P$ is the percentage of power increment corresponding to the $\Delta \mathrm{FoM}_{2}$. For example, in order to improve $\mathrm{FoM}_{2}$ by $10 \mathrm{~dB}$ $\left(\Delta \mathrm{FoM}_{2}=316 \%\right)$ using traditional oversampling method, the power consumption has to increase by $20 \mathrm{~dB}(\Delta P=1000 \%)$. Thus, $\mathrm{FoM}_{3}$ of traditional oversampling method is 2.0 , and so is the $\mathrm{FOM}_{3}$ of basic continuous-time operation [30]. Compared to [17] and [35] which achieve a $F_{0} M_{3}$ of 1.0 and 0.83, respectively, this work achieves a $\mathrm{FOM}_{3}$ of 0.14 . Thus, the BEOSA technique shows the best power efficiency in terms of $\mathrm{FoM}_{3}$ for open-loop MEMS capacitive accelerometer. For the purpose of data integrity, the other parameters measured in the experiments are listed in the table II. The measurement configuration is shown in Fig. 13. The two test capacitors $C_{T}$ are used to inject test charge to CVC to measure the IC sensitivity. The IC sensitivity is then calculated by $V_{R} V_{T O} /$ $V_{T I} C_{T}$, where $V_{T I}$ is the input test voltage and $V_{T O}$ is the output 
TABLE I COMPARISON OF PARASITIC-INDUCED NOISE REDUCTION TECHNIQUES*

\begin{tabular}{|c|c|c|c|c|c|c|}
\hline & $\star$ & {$[17]$} & [9] & {$[35]$} & [39] & {$[40]$} \\
\hline Readout circuit type & \multicolumn{3}{|c|}{ Switched-capacitor } & \multicolumn{3}{|c|}{ Continuous-time } \\
\hline Noise reduction technique & BE-OSA & $\begin{array}{c}\text { Holistic } \\
\text { optimization }\end{array}$ & $\begin{array}{l}\text { Traditional } \\
\text { oversampling }\end{array}$ & $\begin{array}{l}\text { Negative cap. } \\
\text { cancellation }\end{array}$ & \multicolumn{2}{|c|}{$\begin{array}{l}\text { Basic continuous-time } \\
\text { operation }\end{array}$} \\
\hline Para. cap. $C_{P}(\mathrm{pF})$ & 3 & 6 & 3 & 380 & 0.45 & 2.5 \\
\hline Input noise floor $(\mathrm{aF} / \sqrt{\mathrm{Hz}})$ & 0.9 & 613.2 & 54 & 0.08 & 0.38 & 0.85 \\
\hline Bandwidth (Hz) & 10,000 & 30 & 10,000 & 46,000 & 4,000 & 500,000 \\
\hline Supply Current $(\mu \mathrm{A})$ & 45 & 0.13 & 194 & 10,830 & 27 & 88 \\
\hline Supply Voltage (V) & 1.8 & 1.0 & 1.8 & 5.0 & 1.2 & 2.5 \\
\hline Input range $(\mathrm{fF})$ & 10 & 9,500 & - & 6.9 & 48 & 1,800 \\
\hline IC Process (nm) & 180 & 180 & 180 & - & 130 & 65 \\
\hline $\mathrm{FoM}_{2}(\mathbf{f J})$ & 243 & 2,416 & 62,856 & 53 & 435 & 104 \\
\hline $\mathrm{FoM}_{3}$ & 0.14 & 1.0 & 2.0 & $\mathbf{0 . 8 3}$ & 2.0 & 2.0 \\
\hline
\end{tabular}

*All the results of this work are tested under room temperature $\left(27^{\circ} \mathrm{C}\right)$.

voltage excited by $V_{T I}$. By measuring the output voltage change of the readout circuit when $\pm 1 \mathrm{~g}$ acceleration is applied to the accelerometer, the system sensitivity of the accelerometer is obtained. The sensor sensitivity is calculated by dividing the system sensitivity by the IC sensitivity. The acceleration noise is calculated by dividing the input noise floor of CVC (shown in table I) by the sensor sensitivity.

TABLE II PARAMETERS SUMMARY

\begin{tabular}{ll|ll}
\hline \multicolumn{1}{c}{ Parameters } & \multicolumn{1}{c}{ Value } & \multicolumn{1}{c}{ Parameters } & \multicolumn{1}{c}{ Value } \\
\hline IC sensitivity & $90 \mathrm{mV} / \mathrm{fF}$ & Sensor sensitivity & $1 \mathrm{fF} / \mathrm{g}$ \\
Acc. noise & $0.9 \mathrm{mg} / \sqrt{\mathrm{Hz}}$ & Full scale range & $\pm 8 \mathrm{~g}$ \\
Nonlinearity & $0.5 \% @[0,8 \mathrm{~g}]$ & Sampling freq. & $1 \mathrm{MHz}$ \\
\hline \hline
\end{tabular}

\section{CONCLUSION}

The BE-OSA technique is proposed in this paper to reduce the noise floor of readout circuit for MEMS capacitive accelerometer while achieving high power efficiency. The noise floor of readout circuit is dominated by the thermal noise charge from parasitic capacitance in the input terminals of the front-end CVC. Traditional oversampling method can reduce the noise floor at the cost of the increased power consumption which is often too high to be accepted for IoT applications. The BE-OSA readout technique sacrifices the CVC's DT bandwidth rather than power consumption to improve sampling frequency and then compensates the DT bandwidth with negligible increase of power consumption. As a result, the noise floor of SC CVC is significantly reduced while system bandwidth loss and power consumption increment are minimized, i.e., high power efficiency is achieved. The SC CVC based on BE-OSA readout technique is demonstrated in a readout circuit fabricated with a commercial $0.18 \mu \mathrm{m}$ BCD process and tested with a femto-farad MEMS capacitive accelerometer. The measurement results show that compared to the readout circuit without BE-OSA circuits, the readout circuit with BE-OSA circuits reduces the noise floor from $2.5 \mathrm{aF} / \sqrt{\mathrm{Hz}}$ to $0.9 \mathrm{aF} / \sqrt{\mathrm{Hz}}$. Compared with the other similar works, the proposed readout circuit achieves the best power efficiency in terms of both the absolute power efficiency $\left(\mathrm{FoM}_{2}=243 \mathrm{fJ}\right)$ among the switchedcapacitor readout circuits and the relative power efficiency $\left(\mathrm{FoM}_{3}=0.14\right)$.

\section{REFERENCES}

[1] Althoff, Tim, et al. "Large-scale physical activity data reveal worldwide activity inequality." Nature, vol. 547, no. 7663, pp.336-339, Jul. 2017.

[2] Y. Kamada, A. Isobe, T. Oshima, Y. Furubayashi, T. Ido and T. Sekiguchi, "Capacitive MEMS Accelerometer with Perforated and Electrically Separated Mass Structure for Low Noise and Low Power," J. Microelectromech. Syst., vol. 28, no. 3, pp. 401-408, June 2019.

[3] LIS3DH Datasheet. 3-axis MEMS high-performance, ultra-low-power accelerometer. STMicroelectronics. Rev.2. 2016.

[4] AXDL355 Datasheet. Low Noise, Low Drift, Low Power, 3-Axis MEMS Accelerometers. Analog Devices. 2018.

[5] J. I. Seeger and B. E. Boser, "Charge control of parallel-plate, electrostatic actuators and the tip-in instability," J. Microelectromech. Syst., vol. 12, no. 5, pp. 656-671, Oct. 2003.

[6] V. P. Petkov, G. K. Balachandran and J. Beintner, "A Fully Differential Charge-Balanced Accelerometer for Electronic Stability Control," IEEE J. Solid-State Circuits, vol. 49, no. 1, pp. 262-270, Jan. 2014.

[7] B. V. Amini and F. Ayazi, "A 2.5-V 14-bit $\Delta \Sigma$ CMOS SOI capacitive accelerometer," IEEE J. Solid-State Circuits, vol. 39, no. 12, pp. 24672476, Dec. 2004.

[8] H. Ha, D. Sylvester, D. Blaauw and J. Sim, "A 160nW 63.9fJ/conversionstep capacitance-to-digital converter for ultra-low-power wireless sensor nodes," 2014 IEEE International Solid-State Circuits Conference Digest of Technical Papers (ISSCC), San Francisco, CA, 2014, pp. 220-221.

[9] L. Zhong, X. Lai and D. Xu, "Oversampling Successive Approximation Technique for MEMS Differential Capacitive Sensor," IEEE J. SolidState Circuits, vol. 53, no. 8, pp. 2240-2251, Aug. 2018.

[10] M. Lemkin and B. E. Boser, "A three-axis micromachined accelerometer with a CMOS position-sense interface and digital offset-trim electronics," IEEE J. Solid-State Circuits, vol. 34, no. 4, pp. 456-468, Apr. 1999.

[11] M. Paavola et al., "A Micropower $\Delta \Sigma$-Based Interface ASIC for a Capacitive 3-Axis Micro-Accelerometer," IEEE J. Solid-State Circuits, vol. 44, no. 11, pp. 3193-3210, Nov. 2009.

[12] J. Zhao, et al., "A 0.23- g Bias Instability and 1-ug/ $\sqrt{ } \mathrm{Hz}$ Acceleration Noise Density Silicon Oscillating Accelerometer With Embedded Frequency-to-Digital Converter in PLL," IEEE J. Solid-State Circuits, vol. 52, no. 4, pp. 1053-1065, April 2017.

[13] S. Wang et al., "A Power efficient Capacitive Read-Out Circuit with Parasitic-Cancellation for MEMS Cochlea Sensors," IEEE Trans. Biomed. Circuits Syst., vol. 10, no. 1, pp. 25-37, Feb. 2016.

[14] L. Zhong, X. Lai, D.xu, et al., "Capacitive Touch Panel With Low Sensitivity to Water Drop Employing Mutual-Coupling Electrical Field Shaping Technique," IEEE Trans. Circuits Syst. I, Reg. Papers, vol. 66, no. 4, pp. 1393-1404, April 2019.

[15] Pertijs M.A.P., Tan Z. "Power efficient Capacitive Sensor Interfaces.", in: Nyquist AD Converters, Sensor Interfaces, and Robustness. Springer, New York, NY. 2013.

[16] T. He and G. C. Temes, "System-level noise filtering and linearization," 2018 IEEE Custom Integrated Circuits Conference (CICC), San Diego, CA, 2018, pp. 1-8.

[17] M. M. Ghanbari, et.al. "An Power efficient Miniaturized Intracranial Pressure Monitoring System," IEEE J. Solid-State Circuits, vol. 52, no. 3, pp. 720-734, Mar. 2017. 
[18] A. Far, "Class AB amplifier with noise reduction, speed boost, gain enhancement, and ultra-low power," IEEE Latin American Symposium on Circuits \& Systems (LASCAS), Puerto Vallarta, 2018, pp. 1-4.

[19] M. Jang, et.al. "A $55 \mu \mathrm{W}$ 93.1dB-DR 20kHz-BW single-bit CT $\Delta \Sigma$ modulator with negative R-assisted integrator achieving 178.7dB FoM in 65nm CMOS," Symposium on VLSI Circuits, Kyoto, 2017, pp. C40-C41.

[20] S. Cho, S. Kim, S. Lim and K. Baek, "Capacitive proximity sensor with negative capacitance generation technique," Electronics Letters, vol. 48, no. 22, pp. $1409-1411$, Oct. 2012.

[21] R. Wu, et.al, "A 20-b $\pm 40-\mathrm{mV}$ Range Read-Out IC With 50-nV Offset and $0.04 \%$ Gain Error for Bridge Transducers," IEEE J. Solid-State Circuits, vol. 47, no. 9, pp. 2152-2163, Sept. 2012.

[22] L. Wang, P. Ku, P. Ko, C. Chung and L. Lu, "A 40.4-dB Range, 0.73-dB Step And 0.07-dB Error Programmable Gain Amplifier Using Gain Error Shifting Technique," IEEE Trans. Circuits Syst. II, Exp. Briefs. 2019.

[23] Yoshizawa, Hirokazu, and G. C. Temes, "Switched-capacitor track-andhold amplifier with low sensitivity to op-amp imperfections," IEEE Trans. Circuits Syst. I, Reg. Papers, vol. 54, no. 1, pp. 267-270, Jan. 2007.

[24] O. A. Hafiz, X. Wang, P. J. Hurst and S. H. Lewis, "Immediate Calibration of Operational Amplifier Gain Error in Pipelined ADCs Using Extended Correlated Double Sampling," IEEE J. Solid-State Circuits, vol. 48, no. 3, pp. 749-759, March 2013.

[25] B. R. Gregoire and U. Moon, "An Over-60 dB True Rail-to-Rail Performance Using Correlated Level Shifting and an Opamp With Only 30 dB Loop Gain," IEEE J. Solid-State Circuits, vol. 43, no. 12, pp. 26202630, Dec. 2008.

[26] T. Hung and T. Kuo, "A 75.3-dB SNDR 24-MS/s Ring Amplifier-Based Pipelined ADC Using Averaging Correlated Level Shifting and Reference Swapping for Reducing Errors From Finite Opamp Gain and Capacitor Mismatch," IEEE J. Solid-State Circuits, vol. 54, no. 5, pp. 1425-1435, May 2019.

[27] J. Shiah and S. Mirabbasi, A 5-V 290- $\mu$ W Low-Noise Chopper-Stabilized Capacitive-Sensor Readout Circuit in 0.8- $\mu \mathrm{m}$ CMOS Using a CorrelatedLevel-Shifting Technique," IEEE Trans. Circuits Syst. II, Exp. Briefs, vol. 61, no. 4, pp. 254-258, April 2014.

[28] L. L. Lewyn, T. Ytterdal, C. Wulff and K. Martin, "Analog Circuit Design in Nanoscale CMOS Technologies," Proc. IEEE, vol. 97, no. 10, pp. 1687-1714, Oct. 2009.

[29] X. Jiang et al., "Digitally-assisted analog and analog-assisted digital design techniques for a $28 \mathrm{~nm}$ mobile System-on-Chip," European Solid State Circuits Conference (ESSCIRC), Venice Lido, 2014, pp. 475-478.

[30] Murmann B. "Thermal Noise in Track-and-Hold Circuits: Analysis and Simulation Techniques", IEEE Solid State Circuits Mag., vol.4, no.2, pp.46-54, 2012.

[31] R. Kapusta et al., "A 4-channel 20-to300 Mpixel/s analog front-end with sampled thermal noise below kT/C for digital SLR cameras," 2009 IEEE International Solid-State Circuits Conference (ISSCC), San Francisco, CA, 2009, pp. 42-43,43a.

[32] R. Kapusta, H. Zhu and C. Lyden, "Sampling Circuits That Break the kT/C Thermal Noise Limit," IEEE J. Solid-State Circuits, vol. 49, no. 8, pp. 1694-1701, Aug. 2014.

[33] B. D. Sahoo and A. Inamdar, "Thermal-Noise-Canceling SwitchedCapacitor Circuit," IEEE Trans. Circuits Syst., II, Exp. Briefs, vol. 63, no. 7, pp. 628-632, July 2016.

[34] I. Akita, T. Okazawa, Y. Kurui, A. Fujimoto and T. Asano, "A 181nW $970 \mathrm{ug} / \sqrt{\mathrm{Hz}}$ Accelerometer Analog Front-End Employing Feed forward Noise Reduction Technique," 2018 IEEE Symposium on VLSI Circuits, Honolulu, HI, 2018, pp. 161-162.

[35] S. Jeon, et.al, "A Parasitic Insensitive Catheter-Based Capacitive Force Sensor for Cardiovascular Diagnosis," IEEE Trans. Biomed. Circuits Syst., vol. 12, no. 4, pp. 812-823, Aug. 2018.

[36] A. Merassi, B. Murari, and S. Zerbini, "Micro-electromechanical structure with self-compensation of the thermal drifts caused by thermomechanical stresses," U.S. Patent 7520171 B2, Jan. 29, 2009.

[37] Quinn, Patrick J, Roermund, Arthur H. M. Van. "Switched-Capacitor Techniques For High-Accuracy Filter And ADC Design”, Springer, 2007.

[38] Kofi A. A. Makinwa, etc. Low-Power Analog Techniques, Sensors for Mobile Devices, and Energy Efficient Amplifiers. Part III. pp. 253-296. Springer. 2018.

[39] M. De Matteis, A. Pezzotta, M. Sabatini, et.al. "A 90 uW continuous-time front-end with 10b SAR-ADC for capacitive MEMS accelerometers." Analog Integr Circ Sig Process, vol.92, pp.453-465, Jul 2017.

[40] G. Scotti, S. Pennisi, P. Monsurr`o, and A. Trifiletti, "88- $\mu$ A 1-MHz stray insensitive CMOS current-mode interface IC for differential capacitive sensors," IEEE Trans. Circuits Syst. I: Regular Papers, vol. 61, no. 7, pp.
1905-1915, Jul. 2014.

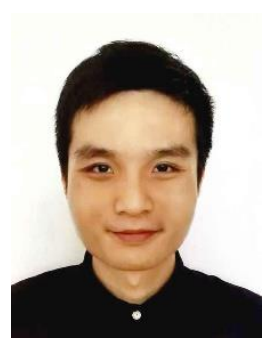

Longjie Zhong (SM'18) received the BSc degree in Biomedical Engineering and $\mathrm{PhD}$ degree in Electronic Science \& Technology from Xidian University, Xi' an, China, in 2012 and 2019. He is also a Visiting Student of Nanyang technological university, Singapore, in 2018. In December 2019, he joined the Southeast University, Nanjing, China and at present he is an Assistant Professor. His research interests focuses on low power, high efficiency ASIC for IoT sensing.

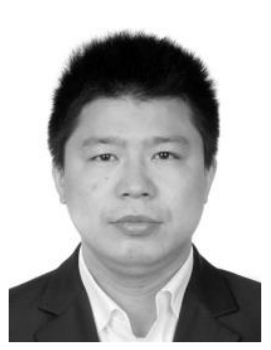

Jun Yang (M'13) received the BSc and $\mathrm{PhD}$ degrees in Electronic Engineering from Southeast University, Nanjing, China, in 1999 and 2004, respectively. He is currently a Professor with the National ASIC System Engineering Research Center, Southeast University. His current research focuses on low power, low voltage (near threshold) ASIC design.

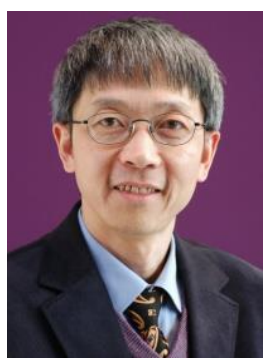

Donglai Xu (M'99-SM'06) received the BSc and MSc degrees in Electronic Engineering from Xidian University, China in 1985 and 1990, respectively, and the $\mathrm{PhD}$ degree in Electronic and Electrical Engineering from the University of Bradford, UK in 1999. Since 1998, Dr Xu has been with Teesside University, UK, where he is a Reader in Electronic Engineering. His research interests include video signal processing, circuits and systems design, VLSI and organic electronic devices. Since 2013, Dr Xu is also a Visiting Professor of Wuhan Polytechnic University, China.

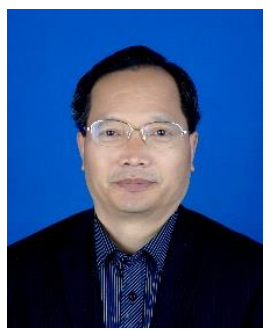

Xinquan Lai (M'18) received the BSc and MSc degrees from School of Electronic Engineering, Xidian University, Xi'an, China, in 1987, 1993 respectively, and he received Ph.D. degree from School of Computer, Northwestern Polytechnical University, Xi'an, China, in 1998. He is currently a professor in the School of Electronic Engineering at Xidian University. His main research interests include mixed-signal ASIC for IoT sensing. 